\title{
Hypoparathyroidism, Retardation, and Dysmorphism Syndrome: Impaired Early Growth and Increased Susceptibility to Severe Infections Due to Hyposplenism and Impaired Polymorphonuclear Cell Functions
}

\author{
ELI HERSHKOVITZ, ILIA ROZIN, YEHUDA LIMONY, HAIM GOLAN, NURIT HADAD, RAFAEL GORODISCHER, \\ AND RACHEL LEVY
}

\begin{abstract}
Pediatric Endocrinology Unit [E.H., I.R., Y.L.], Institute of Nuclear Medicine [H.G.], Department of Clinical Biochemistry [N.H., R.L.], Infectious Diseases Laboratory, Pediatrics Department [R.G.], Soroka Medical University Center and the Faculty of Health Sciences, Ben-Gurion University of the Negev, Beer-Sheva 84101, Israel
\end{abstract}

\begin{abstract}
Hypoparathyroidism, retardation, and dysmorphism (HRD) syndrome is the first reported disease caused by a defect in the tubulin folding and assembly pathway. We aimed to summarize our experience with a cohort of patients with HRD, analyze their growth, and evaluate patients' polymorphonuclear cell (PMN) functions. The records of $22 \mathrm{HRD}$ patients in a single medical center were reviewed. Growth during infancy and early childhood were analyzed by the Infancy-Childhood-Puberty (ICP) growth model. PMN functions were compared with healthy controls. Twelve patients died and many hospitalizations due to infections and convulsions were recorded. Growth measurements, expressed as weight and height SD scores in boys at a mean age of $4 \mathrm{y}$ were $-13.1 \pm 3.8$ and $-8.7 \pm 1$ and $-16.6 \pm$ 4.4 and $-9.5 \pm 2.4$, respectively, in girls at a mean age of $6.4 \mathrm{y}$. Chemotactic migration, random migration, and phagocytosis of PMN from HRD patients were significantly lower than that of PMN from healthy controls. No significant differences were found in superoxide production of PMN from patients compared with controls. Functional hyposplenism has been demonstrated in most of the studied patients. The defect in the tubulin folding and assembly pathway, previously described in HRD, has grave consequences on growth and PMN functions. (Pediatr Res 62: 505-509, 2007)
\end{abstract}

$\mathrm{T}^{2}$ he syndrome of hypoparathyroidism, retardation, and dysmorphism (HRD) (MIM 241410) consists of permanent congenital hypoparathyroidism, severe prenatal and postnatal growth retardation, and profound developmental delay. The patients are susceptible to severe infections including lifethreatening pneumococcal infections, especially during infancy [see reference 1 for a detailed review].

HRD syndrome is the first reported disease caused by a defect in the tubulin folding and assembly pathway due to mutations of the tubulin cofactor $\mathrm{E}$ (TBCE) gene that encodes a chaperone required for $\alpha$ - and $\beta$-tubulin dimerization and microtubule (MT) polymerization (2). MT metabolism performs one of the leading roles in regulating various features of the cytoskeleton, which plays a major role in many cell processes. As an active system, the cytoskeleton is continu-

Received January 31, 2007; accepted May 29, 2007.

Correspondence: Eli Hershkovitz, M.D., Pediatric Endocrinology and Metabolic Unit, Soroka Medical University Center, P.O. Box 151, Beer Sheva 84101, Israel; e-mail: elih@bgu.ac.il ously changing its characteristics, in particular constantly building and destroying the opposite ends of polymers forming its structure.

Professional phagocytes, e.g., neutrophils and macrophages, play an important role in our innate defense against invading pathogens, in the resolution of inflammation, and in the general maintenance of tissue homeostasis $(3,4)$. These functions are highly dependent on the ability of these cells to internalize particles and on other events that are coupled to or triggered by phagocytosis (e.g., secretion, oxidative burst). MTs influence cell surface function in polymorphonuclear cell (PMN) (5). We hypothesized that PMN of HRD patients could have impaired functions due to the MT assembly defect in these patients.

In this report, we summarize our long-term (15 y) experience with a relatively large number of HRD patients in a single medical center, and characterize patients' growth during infancy and early childhood. In addition, we aimed to explore possible mechanisms for the observed increased susceptibility of HRD patients to infections by evaluating their PMN and splenic functions.

\section{PATIENTS AND METHODS}

Subjects. We reviewed the medical records of all patients with HRD syndrome followed at Soroka University Medical Center. All the patients were homozygous for the del52-55 mutation of the TBCE gene, as previously described (2).

Our study was approved by the ethics committee of Soroka Medical University center. Written informed consent was obtained from the patients' and controls' parents for the participation of their children in the immunologic investigations.

General. Clinical and growth data were collected retrospectively according to a designed protocol.

Growth. Birth weight, birth length (or, where this was unavailable, length measured within 1 mo after birth), and subsequent growth data including the latest available weight and height measurements were recorded. SD scores (SDS) of the patients' weight and height were calculated by using the Growth

Abbreviations: FMLP, formyl-methionyl-leucyl-phenylalanine; HRD, hypoparathyroidism, retardation, and dysmorphism; ICP, Infancy-ChildhoodPuberty; MT, microtubule; OZ, opsonized zymosan; PMA, phorbol myristate acetate; PMN, polymorphonuclear (cells); TBCE, tubulin cofactor E 
Analyzer (version 3.0) program applying the 2000 U.S. National Center for Health Statistics growth charts. These growth charts were found to be adequate for growth assessment of Israeli children in the absence of local growth charts (6).

In addition, linear growth during the first three years of life was characterized by applying the Infancy Childhood-Puberty (ICP) growth model. The model "breaks down" linear growth mathematically into three additive components: Infancy (I), Childhood (C), and Puberty (P) (7). The principal advantage of this model over the common growth curves is that it is a longitudinal description of growth rather than cross-sectional. While the usual growth curves are limited to descriptions of height that is not shorter than the 3rd percentile of a normal population, this model enables the examination of the path of growth of very short patients: a clear advantage in HRD patients. To analyze the path of height growth of HRD patients, for each child's measurements we have drawn a "best fitted ICP curve" using a method described elsewhere (8). By this method, each child's path of growth was characterized by two parameters: the height SDS (expressed as deviation in SDS from the I component and calculated according to ICP equations) and the time of onset of the C component.

Spleen Scintigraphy. Tc-99m-colloid Phytate scintigraphy was performed to evaluate splenic function. Scanning was performed 15-30 min following i.v. injection of $1 \mathrm{mCi}$ of the Tc-99m-labeled colloid. Usually, $80-90 \%$ of the injected colloid is taken by the Kupffer cells in the liver and $5-10 \%$ is taken by the reticuloendothelial cells in the spleen. The rest is absorbed by the reticuloendothelial system in the bone marrow (9). Planar images of the liver and spleen were taken in anterior, posterior, left and right lateral, left and right posterior, and anterior oblique views. Spleen uptake was calculated using the geometric mean method from the anterior and posterior views.

Preparation of neutrophils. Neutrophils, at $95 \%$ purity, were obtained by Ficoll-Hypaque centrifugation, dextran sedimentation, and hypotonic lysis of erythrocytes as previously described (10) from 11 patients and 11 age- and sex-matched healthy controls.

Superoxide Anion Measurements. The production of superoxide anion $\left(\mathrm{O}_{2}^{-}\right)$by neutrophils was measured as the superoxide dismutase-inhibitable reduction of acetyl ferricytochrome $\mathrm{c}$ by the microtiter plate technique, as previously described (10) by unstimulated or stimulated neutrophils (by 5 $\mathrm{ng} / \mathrm{mL}$ phorbol myristate acetate (PMA), $1 \mathrm{mg} / \mathrm{mL}$ opsonized zymosan (OZ), or $5 \times 10^{-7} \mathrm{M}$ FMLP).

OZ was prepared as follows: $20 \mathrm{mg}$ zymosan was incubated with $1 \mathrm{~mL}$ of pooled human serum (lipopolysaccharide-free) for $1 \mathrm{~h}$ at $37^{\circ} \mathrm{C}$ and washed three times with Hanks' balanced salt solution (HBSS) buffer.

Chemotaxis. Chemotaxis was assessed on agarose plates as previously described (10). A series of three wells, $2.4 \mathrm{~mm}$ in diameter and spaced $2.4 \mathrm{~mm}$ apart, were formed. The center well of each three-well series received a $10-\mu \mathrm{L}$ aliquot of the $5 \times 10^{5}$ neutrophil suspension, the outer well received $10 \mu \mathrm{L}$ of FMLP $\left(10^{-7} \mathrm{M}\right)$, and the inner well $10 \mu \mathrm{L}$ of HBSS. The dishes were subsequently incubated at $37^{\circ} \mathrm{C}$ in a humidified atmosphere containing $5 \%$ $\mathrm{CO}_{2}$ in air for $2 \mathrm{~h}$. The random and chemotactic migration toward FMLP was measured under a light microscope after fixation and Wright-Giemsa staining.

Phagocytosis. Neutrophils $\left(5 \times 10^{6} / \mathrm{mL}\right)$ were suspended in HBSS and incubated at $37^{\circ} \mathrm{C}$ for $15 \mathrm{~min}$ with $5 \mu \mathrm{L}$ zymosan $(1 \mathrm{mg} / \mathrm{mL})$ opsonized by pooled human serum. Phagocytosis was determined in a smeared sample after Wright-Giemsa staining as described earlier (10).

Statistical Analysis. Independent $t$ test (two-tailed) was applied in the comparison of growth parameters, as expressed in SDS, between boys and girls.

Splenic scintigraphy results of our HRD patients were compared with the normal pediatric data using the one sample $t$ test (two tailed).

Statistical evaluation of the PMN function results was performed using independent $t$ tests. Results are expressed as mean \pm SEM.

\section{RESULTS}

Twenty-two (12 F/10 M) HRD patients were followed in our center during 1990-2005. Their average age was 5.3 y (0.4-14.5). All patients were born at term to consanguineous Bedouin families from several clans.

The causes of the patients' multiple hospitalizations (203) were as follows: infections (65\%) including pneumococcal meningitis and bacteremia, convulsions due to hypocalcemia or epilepsy (19\%), respiratory distress (9\%), and other causes (7\%). The patients were treated with vitamin D and calcium supplements, with thiazides added in some patients. Prophy- lactic antibiotic therapy (once daily amoxicillin) and pneumococcal vaccination were introduced in 1998.

Twelve patients $(55 \%)$ died, mostly before the age of $2 \mathrm{y}$. Six succumbed to sepsis or sepsis-like events including three with culture-proven pneumococcal sepsis. One patient died during cardiac arrhythmia and a 7-y-old female patient died due to unexplained progressive interstitial lung disease. Four patients died outside the hospital from unknown cause.

Growth. All children suffered from intrauterine growth retardation (IUGR) with a resultant low birth weight and short birth length. Mean birth weight was $2100 \pm 200 \mathrm{~g}(-2.2 \pm$ $0.25 \mathrm{SDS})$ in boys and $1970 \pm 450 \mathrm{~g}(-2.6 \pm 0.7 \mathrm{SDS})$ in girls. Mean birth length was $44.7 \pm 3.3 \mathrm{~cm}(-5.1 \pm 1.27$ SDS $)$ in boys and $44.6 \pm 2.75 \mathrm{~cm}(-4.7 \pm 1.7 \mathrm{SDS})$ in girls.

Analysis of growth in our patients by the ICP model revealed that during the first year of life, linear growth followed a path of growth that, although very short, coincided with the first component (I) of the ICP model. However, further decrease in linear growth was observed during the second year of life (Fig. 1).

Growth analysis of the path of growth by the ICP model revealed a markedly delayed appearance of the childhood component that normally occurs between 6 and 12 mo of age, when the infancy component markedly decelerates (7). In HRD patients, the appearance of the $\mathrm{C}$ component occurred at the age of $17.6 \pm 5.6 \mathrm{mo}$ in boys and $19.7 \pm 6 \mathrm{mo}$ in girls.

The latest available growth measurements, expressed as weight and height SDS, in boys at a mean age of $4 \mathrm{y}$ (0.4-9.76) were $-13.1 \pm 3.8$ and $-8.7 \pm 1$, respectively. In girls, the latest available weight and height SDS at a mean age of $6.4 \mathrm{y}(1.3-14)$ were $-16.6 \pm 4.4$ and $-9.5 \pm 2.4$, respectively. BMI SDS or weight for length SDS (in patients younger than $3 \mathrm{y}$ ) was below -2 in all the patients except three. The differences in growth between boys and girls were not significant.

Splenic function. Tc-99m-colloid phytate scintigraphy was performed in 11 patients and revealed very low uptake of the tracer in the patients' spleen, with a mean of $2.1 \pm 0.7 \%$. Only two patients had uptake within the normal range (above 5\%) and one subject had no uptake at all. This abnormally low uptake indicates functional hyposplenism in most of the patients and functional asplenia in the severe cases. Analytical statistics aided us in showing that this is a true phenomenon. In comparison to the expected uptake of $5-10 \%$ in normal children (9), the HRD patients showed a lower uptake in the spleen $(\mathrm{t}=-7.81, d f=10, p<0.001$, mean difference $=-5.4)$. In this comparison, we acted upon the hypothesis that the mean uptake should be $7.5 \%$. Even under more conservative assumptions, (i.e., mean uptake is only 5\%) the mean difference between the HRD patients and normal children was still significant $(\mathrm{t}=-4.19, d f=10, p=0.002)$.

Other investigations. Abnormally low plasma levels of insulin like growth factor $1(<-2 \mathrm{SD}$ below the mean for age and sex) were found in all six patients examined. However, growth hormone deficiency, determined by abnormal response to two formal stimulation tests, was diagnosed in only one patient. 

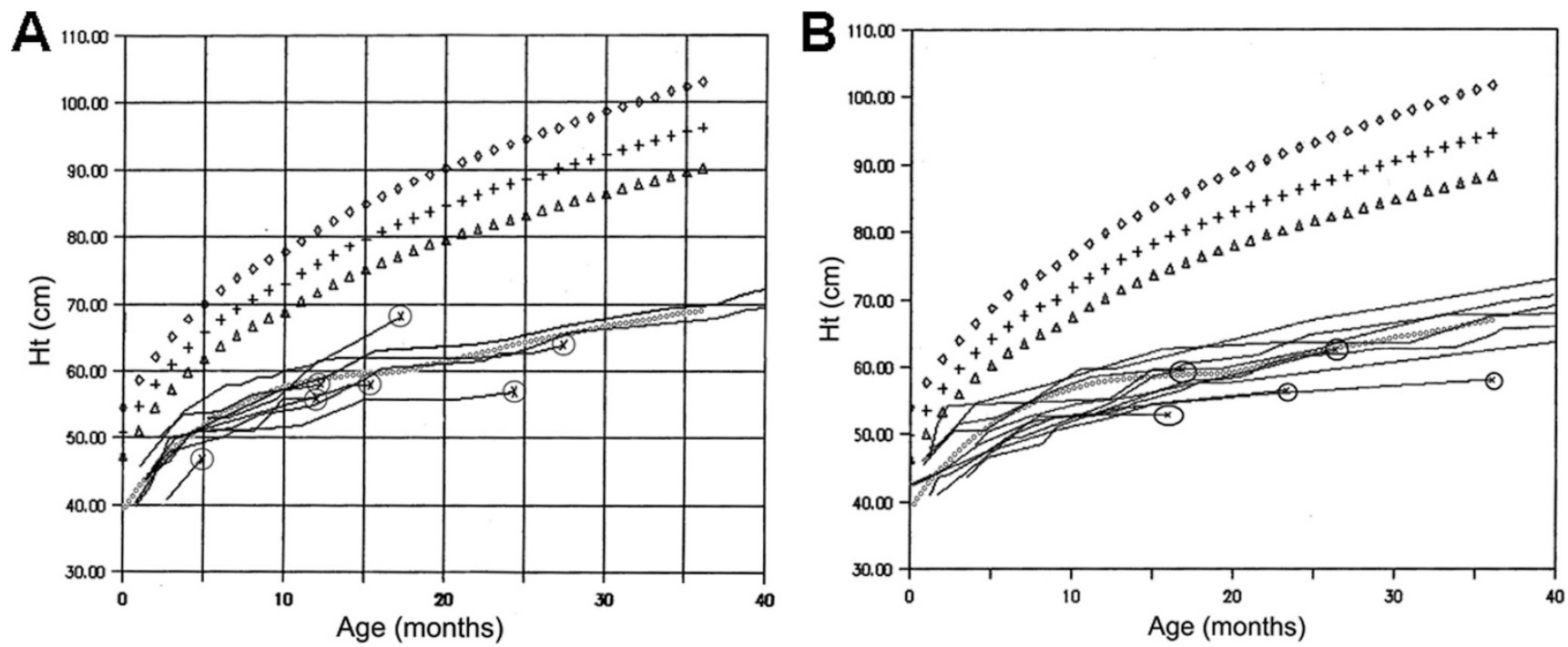

Figure 1. Growth of HRD children according to the ICP model during the first 3 y of life. Upper three lines: growth of normal boys $(A)$ and girls $(B)(\diamond,+2$ SDS; $+=0$ SDS; $\Delta=-2$ SDS). Below are depicted the individual growth lines of HRD boys $(A)$ and girls $(B)$. Average heights of HRD patients $(\bigcirc)$ lines in boys $(A)$ and girls $(B) . \otimes-$ represent the last available height measurements of HRD patients who died later.

Nephrocalcinosis was discovered in 7 out of 31 renal sonograms. No significant cardiac malformations have been found by echocardiography (13 patients). The radiologic ( $\mathrm{x}-$ ray and computed tomography) findings were not specific and were related to the clinical status of the patients at the time of the examinations. Craniosynostosis and brain calcifications were the common findings.

PMN functions. Chemotactic migration toward FMLP of PMN from HRD patients was significantly lower $(p<0.001)$ than that of PMN from healthy controls (Fig. 2A), indicating a severe defect in chemotactic migration in the patients. The mean \pm SEM were $23.3 \pm 2.1$ and $41.5 \pm 0.4 \times 10^{-3} \mathrm{~mm}$ for HRD patients and controls, respectively. There was a clear discrimination with no overlapping in the values between the two groups. In addition, there was a significant difference $(p<$ 0.001 ) in random migration (chemokinesis) between neutrophils from HRD patients and healthy controls (Fig. 2B), with mean \pm SEM of $16.7 \pm 1.9$ and $27.0 \pm 0.85 \times 10^{-3} \mathrm{~mm}$ for HRD patients and controls, respectively. As presented in Figure $3 A$, phagocytosis of $\mathrm{OZ}$ assayed for $15 \mathrm{~min}$ was significantly reduced $(p<0.001)$ in PMN from HRD patients with mean \pm SEM of $60.8 \pm 2 \%$ compared with $96 \pm 3 \%$ in PMN of the healthy controls. Moreover, PMN morphology after phagocytosis is different in the two groups; while PMN from healthy controls were spread out as a result of engulfment of several particles of OZ, most of the PMN from HRD patients were condensed and phagocytized only one or two OZ particles (Fig. 3B). In contrast to chemotaxis and phagocytosis, no significant differences in superoxide production of unstimulated or stimulated neutrophils with OZ, FMLP, or PMA were observed (Fig. 4). The mean \pm SEM of superoxide release by PMN of healthy controls were $4.1 \pm 1.0,10 \pm 0.5$, $5.7 \pm 1.0$, or $28.5 \pm 2.3 \mathrm{nmol} \mathrm{O}_{2} / 10^{6}$ cells $/ \mathrm{min}$, in unstimulated or cells stimulated with OZ, FMLP, or PMA, respectively. The mean \pm SEM of superoxide release by PMN of HRD patients were $4.5 \pm 1.1,9.9 \pm 1.3,5.9 \pm 1.1$, or $24.2 \pm 3.4$
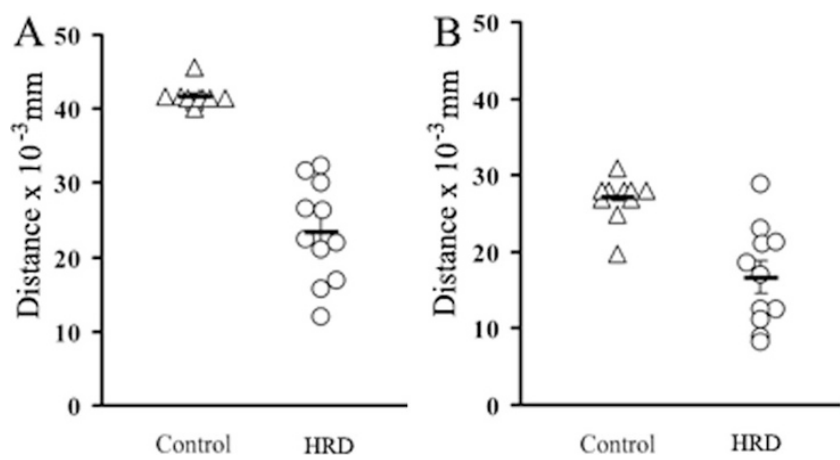

Figure 2. PMN chemotaxis in HRD patients and healthy controls. Chemotactic migration toward FMLP $(A)$ and random migration $(B)$ of PMN from HRD patients and healthy controls. There are significant differences in specific and random migration between the two groups $(p<0.001)$.

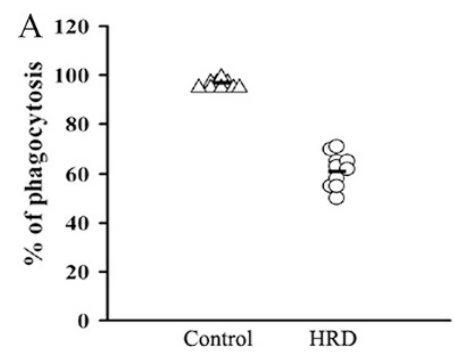

B

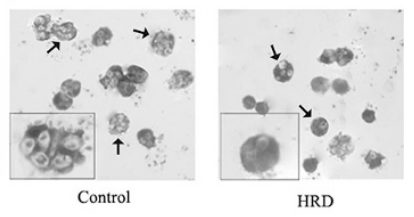

Figure 3. PMN phagocytosis in HRD patients and healthy controls. (A) Phagocytosis of OZ by PMN of HRD patients or healthy controls; significant difference $(p<0.001)$. (B) Wright-Giemsa staining of neutrophils from representative HRD patient (right) and healthy control (left) after 15 min of phagocytosis $(\times 400)$. Arrows show examples of efficient phagocytosis in a healthy control and defective phagocytosis in a HRD patient as shown for a representative cell $(\times 1000)$ in the insets.

nmol $\mathrm{O}_{2} / 10^{6}$ cells $/ \mathrm{min}$, in unstimulated or cells stimulated with OZ, FMLP, or PMA, respectively.

Some patients showed significantly reduced superoxide production stimulated by PMA. These patients also exhibited the most profound defect in chemotaxis and phagocytosis. 


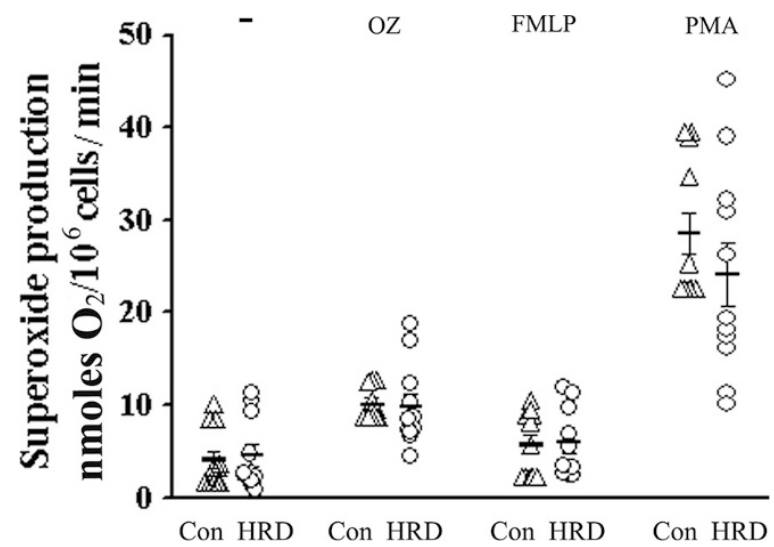

Figure 4. PMN superoxide production in HRD patients and healthy controls. Superoxide production by unstimulated PMN HRD patients $(H R D)$ and healthy controls (Con) and in response to stimulation with $1 \mathrm{mg} / \mathrm{mL} \mathrm{OZ,} 5 \times$ $10^{-7}$ FMLP or $50 \mathrm{ng} / \mathrm{mL}$ PMA.

\section{DISCUSSION}

In this article, we present our experience in a single medical center with the largest number of HRD patients hereto described. The identification of the molecular basis of HRD syndrome enabled us to study the possible effects of the mutated TBCE gene.

Our report confirms the high morbidity and mortality rates of this syndrome. The main causes of the multiple hospitalizations were infections and hypocalcemic convulsions. The observed 55\% mortality rate in our patients due mostly to infections is higher than the reported rate of $25-44 \%$ during infancy and early childhood and may represent a longer period of follow-up and a larger study group in comparison to previous publications (11-14). Recurrent infections and hypocalcemic seizures were the main reported causes of death in some infants but the cause(s) of death remained undetermined in others (11-14). Prophylactic antibiotic therapy and vaccinations including conjugated pneumococcal vaccines are warranted in an attempt to prevent serious infections.

HRD patients are of extremely short stature. We were able to identify two periods of attenuated growth that lead to their short stature. The first was the intrauterine period, which caused the major attenuation. This period of attenuated growth was not followed by "catch-up" growth all along the follow-up period of the study. The second period of attenuated growth took place during the second year of life with a significantly delayed appearance of the $\mathrm{C}$ component.

This delay is responsible for the growth retardation seen after the first year of life, when growth is almost arrested in our patients from about 15 mo of age until the appearance of the $\mathrm{C}$ component. Late appearance of the $\mathrm{C}$ component has been previously described in various states of slow growth in infancy and early childhood such as in Turner syndrome, chronic diarrhea, and celiac disease (15-17).

Since a strong relationship has been described between the shape of the hypertrophic chondrocytes and the rate of longitudinal bone growth in the rat (18) and an important role for microtubules in the control of chondrocyte maturation and hypertrophy has been reported in the chick growth plate (19), we suggest that the pathogenesis of the extreme growth retardation in HRD might be explained by primary impairment in the long bone growth plate function due to the defect in MT assembly and folding. However, these hypothesis should be tested in animal models or pathologic specimens obtained from HRD patients.

An additional impairment of the growth hormone IGF-I axis may also contribute to the growth retardation after infancy, but further studies are required to evaluate this question. Malnutrition, as evidenced by very low BMI or weight for length, and recurrent infections can be implicated in the observed poor growth and low IGF1 levels.

Our study demonstrated that at least two causes might explain the unusual susceptibility of HRD patients to severe infections, namely hyposplenism and abnormal PMN functions. Nine out of 11 subjects had abnormally low Tc (99) uptake by their spleen, indicating functional hyposplenism. Splenic impairment may account for the presence of severe pneumococcal infections.

Of the PMN functions studied, we demonstrated for the first time that directed chemotaxis toward FMLP, random migration, and phagocytosis of OZ were severely defected in the HRD patients (Figs. 2 and 3). In contrast, superoxide production did not differ from that in the healthy controls studied in parallel (Fig. 4). Directional cell migration is a fundamental process in all organisms that is stringently regulated during chemotaxis. Migrating cells have a polarized morphology with an asymmetrical distribution of signaling molecules and the cytoskeleton. MTs are indispensable for the directional migration of certain cells (20). Additionally, they are hollow tubes composed of 13 protofilaments of a- and b-tubulin dimers organized in a headto-tail fashion. MTs are nucleated from their minus ends, which localize predominantly at the MT organizing center. During cell migration, selective stabilization of the plus ends of microtubules enables the MT organizing center to reorient toward the leading edge, resulting in a polarized microtubule array that facilitates cell migration (20).

The gene TBCE, which is mutated in patients with HRD, encodes a chaperone required for the folding of $\alpha$-tubulin and its heterodimerization with $\beta$-tubulin. We have previously shown that tubulin protein abundance and $\alpha$-tubulin transcript levels are similar in diseased and control lymphoblastoid samples (2). On the other hand, the proportion of precipitated MT-incorporated $\alpha$-tubulin was lower in diseased cells and the MT-network polarity was lost in lymphoblastoid cells and in dermal fibroblasts obtained from HRD patients (2). The severe reduction in PMN migration and phagocytosis in the HRD patients demonstrated in our study is probably due to MT dysfunction resulting from the TBCE defect. Cell polarization, migration, and directional extension of pseudopodia in PMN require the MT cytoskeleton (21). Defective chemotaxis of PMN has been shown to be associated with impaired MT organization in pathologic disorders such as Chediak-Higashi syndrome and glutathione synthetase deficiency, as well as in neonates (22-24).

The reduced phagocytosis of OZ which is mediated by Fcg and complement receptors on PMN membranes is probably also due to MT impairment since MTs have been implicated in 
the phagocytosis process which is responsible for engulfment of the particles (25). In human leukocytes, contact of the opsonized particle with its receptors initiates a rapid MT assembly (26). Numerous studies have shown that MTs are not only remodeled during phagocytosis but are also essential for optimal FcgR-mediated ingestion (27-29). MT disruption reduces pseudo pod dynamics and formation. It is plausible that MTs are required for pseudo pods formation during FCg-R-mediated phagocytosis (30,31). In addition, late phagosomes in neutrophils were found to localize adjacent to the centriole (32), and movement of phagosomes along microtubules was documented directly (33).

The production of superoxides by unstimulated neutrophils or by those stimulated with either OZ, PMA, or FMLP was normal in the HRD patients. These results are in accordance with earlier studies demonstrating that NADPH oxidase activity is independent of microtubule formation. MT disruption in neutrophils or in HL-60 cells treated with colchicine or inhibition of MT assembly by vincristine did not affect superoxide radical production stimulated by Fc receptors, receptors for FMLP, or PMA $(34,35)$.

In conclusion, impaired MT function caused by the mutated TBCE gene in HRD syndrome may contribute to the severe growth retardation and to high susceptibility to infections. Reduced PMN chemotaxis and impaired phagocytosis, in addition to hyposplenism explain this susceptibility to infections.

\section{REFERENCES}

1. Hershkovitz E, Parvari R, Diaz GA, Gorodischer R 2004 Hypoparathyroidismretardation-dysmorphism (HRD) syndrome-a review. J Pediatr Endocrinol Metab 17:1583-1590

2. Parvari R, Hershkovitz E, Grossman N, Gorodischer R, Loeys B, Zecic A, Mortier G, Gregory S, Sharony R, Kambouris M, Sakati N, Meyer BF, Al Aqeel AI, Al Humaidan AK, Al Zanhrani F, Al Swaid A, Al Othman J, Diaz GA, Weiner R, Khan KT, Gordon R, Gelb BD HRD/Autosomal Recessive Kenny-Caffey Syndrome Consortium 2002 Mutation of TBCE causes hypoparathyroidism- retardationdysmorphism and autosomal recessive Kenny-Caffey syndrome. Nat Genet 32:448452

3. Witko-Sarsat V, Rieu P, Descamps-Latscha B, Lesavre P, Halbwachs-Mecarelli L 2000 Neutrophils: molecules, functions and pathophysiological aspects. Lab Invest 80:617-653

4. Henson PM, Henson JE, Fittschen C, Bratton DL, Riches DW 1992 Degranulation and secretion by phagocytic cells. In: Gallin, L Goldstein IM Snyderman R (eds) Inflammation: Basic Principles and Clinical Correlates, 2nd Ed. Raven Press, New York, 511-525

5. Berlin RD, Fera J 1977 Changes in membrane microviscosity associated with phagocytosis: effects of colchicine. Proc Natl Acad Sci U S A 74:1072-1076

6. Goldstein A, Haelyon U, Krolik E, Sack J 2001 Comparison of body weight and height of Israeli schoolchildren with the Tanner and Centers for Disease Control and Prevention growth charts. Pediatrics 108:E108

7. Karlberg J 1989 A biologically-oriented mathematical model (ICP) for human growth. Acta Paediatr Scand Suppl 350:70-94

8. Limony Y, Leiberman E, Hershkovitz E 2004 The Childhood component of the ICP Model is appropriate for growth analysis of short Israeli children. Horm Res 62:119-123
9. Treves ST, Jones AG, Markisz J 1995 Liver and spleen. In: Treves ST (ed) Pediatric Nuclear Medicine. Springer-Verlag, New York, 470-471

10. Liel Y, Rudich A, Nagauker Shriker O, Yermiyahu T, Levy R 1994 Monocyte dysfunction in patients with Gaucher disease: evidence for interference of glucocerebroside with superoxide generation. Blood 83:2646-2653

11. Sanjad SA, Sakati NA, Abu-Obsa YK, Kaddoura R, Milner RD 1991 A new syndrome of congenital hypoparathyroidism, severe growth failure, and dysmorphic features. Arch Dis Child 66:193-196

12. Hershkovitz E, Shalitin S, Levy J, Leiberman E, Weinshtock A, Varsano I, Gorodischer R 1995 The new syndrome of congenital hypoparathyroidism, growth retardation, and developmental delay. a report of six patients. Isr $\mathrm{J}$ Med Sci 31:293-297

13. Richardson RJ, Kirk JM 1990 Short stature, mental retardation and hypoparathyroidism: a new syndrome. Arch Dis Child 65:1113-1117

14. Tahseen K, Khan S, Uma R, Usha R, Al Ghanem MM, Al Awadi SA, Farag TI 1997 Kenny-Caffey syndrome in six Bedouin sibships: autosomal recessive inheritance is confirmed. Am J Med Genet 69:126-132

15. Karlberg J, Albertsson-Wikland K, Nilsson KO, Ritzen EM, Westphal O 1991 Growth in infancy and childhood in girls with Turner's syndrome. Acta Paediatr Scand 80:1158-1165

16. Liu YX, Jalil F, Karlberg J 1998 Risk factors for impaired length growth in early life viewed in terms of the infancy-childhood-puberty (ICP) growth model. Acta Paediatr 87:237-243

17. Karlberg J, Henter JI, Tassin E, Lindblad BS 1988 Longitudinal analysis of infantile growth in children with celiac disease. Acta Paediatr Scand 77:516-524

18. Hunziker EB, Schenk RK 1989 Physiological mechanisms adopted by chondrocytes in regulating longitudinal bone growth in rats. J Physiol 414:55-71

19. Farquharson C, Lester D, Seawright E, Jefferies D, Houston B 1999 Microtubules are potential regulators of growth-plate chondrocyte differentiation and hypertrophy. Bone 25:405-412

20. Watanabe T, Noritake J, Kaibuchi K 2005 Regulation of microtubules in cell migration. Trends Cell Biol 15:76-83

21. Keller H, Niggli V 1995 Effects of cytochalasin D on shape and fluid pinocytosis in human neutrophils as related to cytoskeletal changes (actin, alpha-actinin and microtubules). Eur J Cell Biol 66:157-164

22. Oliver JM 1976 Impaired microtubule function correctable by cyclic GMP and cholinergic agonists in the Chediak-Higashi syndrome. Am J Pathol 85:395-418

23. Oliver JM, Spielberg SP, Pearson CB, Schulman JD 1978 Microtubule assembly and function in normal and glutathione synthetase-deficient polymorphonuclear leukocytes. J Immunol 120:1181-1186

24. Anderson DC, Hughes BJ, Wible LJ, Perry GJ, Smith CW, Brinkley BR 1984 Impaired motility of neonatal PMN leukocytes: relationship to abnormalities of cell orientation and assembly of microtubules in chemotactic gradients. J Leukoc Biol $36: 1-15$

25. Harrison RE, Grinstein S 2002 Phagocytosis and the microtubule cytoskeleton Biochem Cell Biol 80:509-515

26. Burchill BR, Oliver JM, Pearson CB, Leinbach ED, Berlin RD 1978 Microtubule dynamics and glutathione metabolism in phagocytizing human polymorphonuclear leukocytes. J Cell Biol 76:439-447

27. Athlin L, Domellof L, Norberg BO 1985 The phagocytosis of yeast cells by blood monocytes. Effects of therapeutic concentrations of Vinca alkaloids. Eur J Clin Pharmacol 29:471-476

28. Athlin L, Domellof L, Norberg BO 1986 The phagocytosis of yeast cells by monocytes: effects of colchicine, beta-lumicolchicine, and gamma-lumicolchicine. Lymphology 19:130-135

29. Bjermer L, von Schoultz E, Norberg B, Henriksson R 1988 Estramustine inhibits monocyte phagocytosis. Prostate 13:49-55

30. Rosania GR, Swanson JA 1996 Microtubules can modulate pseudopod activity from a distance inside macrophages. Cell Motil Cytoskeleton 34:230-245

31. Nabi IR 1999 The polarization of the motile cell. J Cell Sci 112:1803-1811

32. Hoffstein S, Goldstein IM, Weissmann G 1977 Role of microtubule assembly in lysosomal enzyme secretion from human polymorphonuclear leukocytes. J Cell Biol 73:242-256

33. Blocker A, Griffiths G, Olivo JC, Hyman AA, Severin FF 1998 A role for microtubule dynamics in phagosome movement. J Cell Sci 111:303-312

34. Reibman J, Haines KA, Gude D, Wissmann G 1991 Differences in signal transduction between $\mathrm{Fc}$ gamma receptors (Fc gamma RII, Fc gamma RIII) and FMLP receptors in neutrophils. Effect of colchicine on pertussis toxin sensitivity and diacylglycerol formation. J Immunol 146:988-996

35. Vrba J, Modriansky M 2004 N-formyl-Met-Leu-Phe-induced oxidative burst in DMSO-differentiated HL-60 cells requires active Hsp90, but not intact microtubules. Biomed Pap Med Fac Univ Palacky Olomouc Czech Repub 148:141-144 